\title{
QUEEN'S
UNIVERSITY
BELFAST
}

\section{Directional Modulation Transmitter Synthesis using Particle Swarm Optimization}

Ding, Y., \& Fusco, V. (2013). Directional Modulation Transmitter Synthesis using Particle Swarm Optimization. In 2013 Loughborough Antennas \& Propagation Conference (LAPC) (pp. 500-503). Institute of Electrical and Electronics Engineers Inc.. https://doi.org/10.1109/LAPC.2013.6711950

Published in:

2013 Loughborough Antennas \& Propagation Conference (LAPC)

Document Version:

Peer reviewed version

Queen's University Belfast - Research Portal:

Link to publication record in Queen's University Belfast Research Portal

\begin{abstract}
Publisher rights
( 2015 IEEE. Personal use of this material is permitted. Permission from IEEE must be obtained for all other uses, in any current or future media, including

reprinting/republishing this material for advertising or promotional purposes, creating new collective works, for resale or redistribution to servers or lists, or reuse of any copyrighted component of this work in other works.
\end{abstract}

\section{General rights}

Copyright for the publications made accessible via the Queen's University Belfast Research Portal is retained by the author(s) and / or other copyright owners and it is a condition of accessing these publications that users recognise and abide by the legal requirements associated with these rights.

Take down policy

The Research Portal is Queen's institutional repository that provides access to Queen's research output. Every effort has been made to ensure that content in the Research Portal does not infringe any person's rights, or applicable UK laws. If you discover content in the Research Portal that you believe breaches copyright or violates any law, please contact openaccess@qub.ac.uk. 


\title{
Directional Modulation Transmitter Synthesis using Particle Swarm Optimization
}

\author{
Yuan Ding and Vincent Fusco \\ The ECIT Institute Queens University of Belfast \\ Belfast, BT3 9DT, UK \\ yding03@qub.ac.uk
}

\begin{abstract}
Phased DM transmitter array synthesis using particle swarm optimization (PSO) is presented in this paper. The PSO algorithm is described in details with key parameters provided for 1-D four-element half-wavelength spaced QPSK DM array synthesis. A DM transmitter array for boresight and $30^{\circ}$ direction secure communications are taken as examples to validate the proposed synthesis approach. The optimization process exhibits good convergence performance and solution quality.
\end{abstract}

Keywords- bit error rate; directional modulation; particle swarm optimization

\section{INTRODUCTION}

As the extensive deployment of wireless networks, we require to be online and share data almost everywhere and everytime. However, this facility often comes at the expense of security due to the broadcast nature of wireless communications [1]. Traditionally the wireless secrecy problem has been handled at the application layer by appropriate encoding for data encryption and conveyance of secret keys to legitimate receivers via private and secured transmissions. However, malicious eavesdroppers can still capture the encrypted information and have a chance to decode it with large amount of computational resources. Unlike these traditional approaches, physical layer security [2], [3] aims to destroy the leaked information contents, potentially intercepted by eavesdroppers, by exploiting the properties of the physical layer, while simultaneously keeping the information contents delivered to legitimate receivers intact.

Recently proposed directional modulation (DM) concept [4]-[9] is a promising technique for physical layer security. In [4] the orthogonality of Walsh waveforms was exploited to generate direction-sensitive pulse position modulation (PPM) signals. In [5], [6] a parasitic DM structure, termed by the authors as near-field direct antenna modulation (NFDAM), was introduced. By combining a direct radiation beam and several scattered beams, reflected by reconfigurable near-field parasitic structures, in the far-field, signal magnitude and phase relationships along unsecured spatial communication directions can be scrambled. In contrast to parasitic DM structures, actively driven DM arrays [7]-[9] can be more synthesis-friendly, since they allow us to link array excitation settings to the far-field patterns, and ultimately to the DM system performance.

In this paper, we link the system bit error rate (BER) performance to the settings of phase shifters, which are placed in each carrier route to actively driven array elements, in such a fashion so as to synthesize and optimize DM transmitter arrays by the particle swarm optimization (PSO) algorithm.

This work was sponsored by the Queen's University of Belfast High Frequency Research Scholarship.
In Section II, a typical phased DM transmitter array is described. In Section III the BER calculation in a DM system for QPSK modulation scheme and the cost function designs are investigated. The PSO algorithm and parameter configurations for four-element QPSK DM array synthesis are presented in Section IV, followed by synthesis results. Finally, summaries and conclusions are drawn in Section V.

\section{PHASED DM TRANSMITTER ARRAYS}

$\mathrm{DM}$ is a transmitter side technology that can project digital symbols with standard modulation mappings in IQ space along a prescribed spatial direction while simultaneously distort the constellation formats of the same digital symbols in all other directions. For example, a QPSK DM system is illustrated in Fig. 1. Along a user specified direction a standard QPSK constellation pattern is formed. Away from the desired direction the constellation is scrambled.

A typical actively driven phased DM array architecture is shown in Fig. 2. Prior to transmission via $N$ antenna elements, amplitude weighted $\left(A_{m n}\right)$ carriers $\left(f_{c}\right)$ are modulated by baseband information data controlled phase shifters whose values are Phase $_{m n}$, where $m(m=1,2, \ldots, M)$ and $n(n=1,2, \ldots$, $N$ ) correspond to the $m^{\text {th }}$ signal symbol and the $n^{\text {th }}$ array element respectively. We assume that each array element has isotropic radiation pattern. Thus the array factor $(A F)$ associated with the $m^{\text {th }}$ signal excitation can be regarded as the

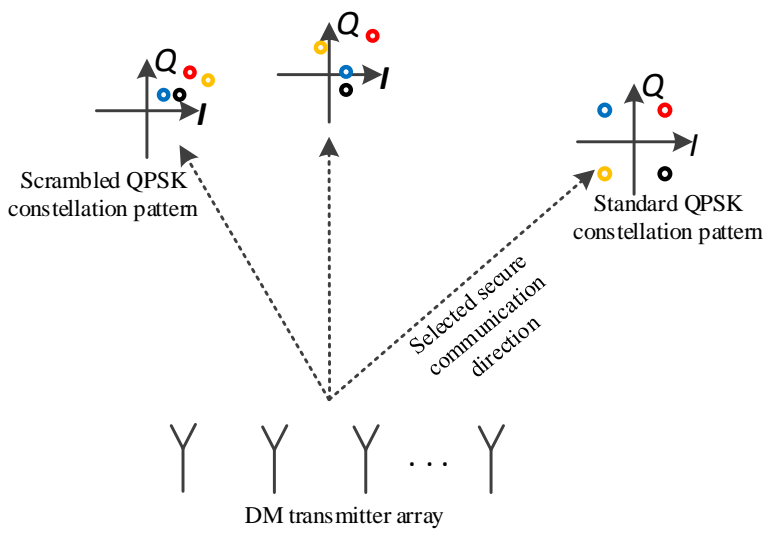

Fig. 1. Illustration of the major properties of a DM QPSK system.

$$
S_{m}=\sum_{n=1}^{N}\left(A_{m n} e^{-j \cdot \text { Phase }_{m n}+j \cdot\left(\bar{k} \cdot \bar{d}_{n}\right)}\right)
$$




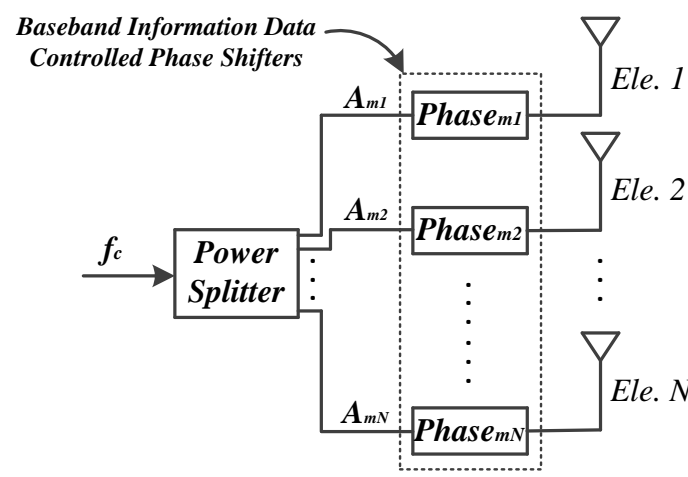

Fig. 2. Typical phased DM array architecture.

$m^{\text {th }}$ received symbol $\left(S_{m}\right)$ in IQ space along each spatial directions, (1). $\vec{k}$ is the wavenumber vector along the spatial transmission direction, and $\vec{d}_{n}$ represents the location vector of the $n^{\text {th }}$ array element relative to the array phase centre.

\section{BER CALCULATION FOR QPSK MODULATION AND COST FUNCTION DESIGNS}

\section{A. BER Calculation for QPSK Modulation}

Once constellation points $S_{m}$ for each unique modulation symbol are obtained via (1), we need to map constellation patterns to the system performance, e.g., BER is used in this paper.

We assume that signals are modulated for QPSK with Gray coding, thus four QPSK symbols ' 11 ', ' 01 ', ' 00 ' and ' 10 ' should lie in the first to the fourth quadrants in IQ space respectively along the direction pre-assigned for transmission. With the capability of scrambling constellation symbols possessed by DM transmitters, four constellation points detected along unselected communication directions no longer form a central symmetric square in IQ space, e.g., a distorted constellation pattern is shown in Fig. 3.

Assume that both legitimate and eavesdroppers are equipped with standard QPSK receivers, in order to decode

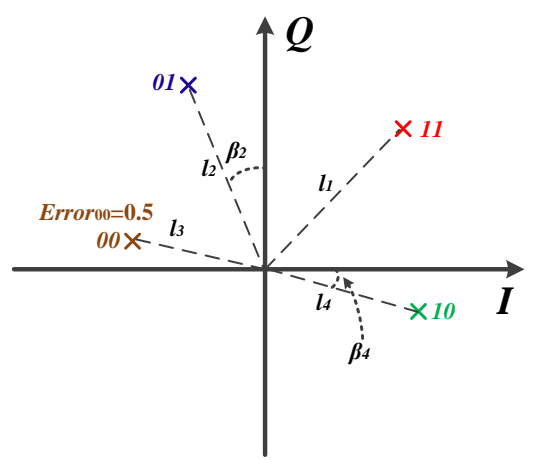

Fig. 3. Illustration of a distorted QPSK constellation pattern in a DM system and parameters of $l_{i}$ and $\beta_{i}$ for BER calculations.

received symbols based on which quadrant the constellation points locate into. For discussion below, the phase of the noiseless symbol ' 11 ' is set as the reference. This means that the received constellation patterns are always rotated to align the phase of the symbol ' 11 ' to $\pi / 4$ in the first quadrant, Fig. 3 . Under such circumstances, BER can be calculated approximately by (2),

$$
\begin{aligned}
& B E R_{D_{-} Q P S K}= \\
& \left.\frac{1}{4} \cdot[\overbrace{Q\left(\sqrt{\frac{l_{1}^{2} \cdot \sin ^{2}(\pi / 4)}{N_{0} / 2}}\right)}^{{\text {Error } r_{11}}}) \text { Error }_{01}+\text { Error }_{00}+\text { Error }_{10}\right]
\end{aligned}
$$

where the Error ${ }_{x y}$ is the bit error rate detected when the symbol ' $x y$ ' is transmitted ( $\{11$ ', '01', '00', ' 10 ' $\}$ ). Under our synchronization assumption, the noiseless symbol ' 11 ' is always phase aligned, thus Error $_{11}$ can be calculated by $Q\left(\sqrt{\frac{l_{1}^{2} \cdot \sin ^{2}(\pi / 4)}{N_{0} / 2}}\right)$ [10]. For the other three symbols, the Error $_{x y}$ can be obtained as $Q\left(\sqrt{\frac{l_{i}^{2} \cdot \sin ^{2}\left(\beta_{i}\right)}{N_{0} / 2}}\right)(i=2,3,4)$ when the noiseless symbol ' $x y$ ' is constrained within its appropriate quadrant. Parameter $\beta_{i}$ is the minimum angle between the symbol vector and the decoding boundary, which overlaps the IQ axes, illustrated in Fig. 3. Otherwise Error Exy $_{x y}$ approximates to 0.5 or 1 depending on which quadrant this distorted noiseless symbol locates into, e.g., if the noiseless symbol ' 00 ' is located into the second (or the first) quadrant, where the symbol ' 01 ' (or ' 11 ') should be, the Error 00 is set to be 0.5 (or respectively 1). It should be noted that when the four constellation points in a DM QPSK system overlay their corresponding standard QPSK symbols, (2) can be expressed as in (3), which is the equation for calculating BER in a standard QPSK modulation system with Gray coding. $d$ is the distance between each two adjacent symbols in IQ space.

$$
B E R_{s_{-} Q P S K}=Q\left(\sqrt{\frac{(d / 2)^{2}}{N_{0} / 2}}\right)
$$

Since constellation points $S_{m}$ are functions of spatial direction $\theta$, BER calculated by (2) is also spatially distributed from $0^{\circ}$ to $180^{\circ}$ with boresight located at $90^{\circ}$.

\section{B. Cost function designs}

In order to carry out optimization in next section, we design a cost function in (4)

$$
V_{c f}=\int_{0}^{\pi} W \cdot\left(B E R_{D_{-} Q P S K}-B E R_{\text {tem }}\right)^{2} d \theta
$$

where $B E R_{\text {tem }}$ is a spatial optimization template, and $W$ is the spatial weights.

In this paper we assume the signal to noise ratio (SNR) along desired communication direction $\theta_{0}$ is $15 \mathrm{~dB}$, so that $\operatorname{BER}\left(\theta_{0}\right)$ can reach $10^{-8}$. 
Taking $90^{\circ}$ communications as an example, we can set $B E R_{\text {tem }}$ to be 1 everywhere other than $10^{-8}$ at boresight and design the weights as in (5). Note (5) how two tapered sections prevent the optimized BER beams from shifting around in space.

$$
W= \begin{cases}1 \quad \theta \in\left[0^{\circ}, 80^{\circ}\right) \cup\left(100^{\circ}, 180^{\circ}\right] \\ 1+\frac{10^{3}-1}{5^{\circ}} \cdot\left(\theta-80^{\circ}\right) & \theta \in\left[80^{\circ}, 85^{\circ}\right) \\ 10^{18} \quad \theta=90^{\circ} & \theta \in\left(95^{\circ}, 100^{\circ}\right] \\ 10^{3}+\frac{1-10^{3}}{5^{\circ}} \cdot\left(\theta-95^{\circ}\right) & \\ 0 \quad \text { otherwise } & \end{cases}
$$

\section{PARTICLE SWARM OptimizATION ALGORITHM AND DM SYNTHESIS RESULTS}

Through (4) a link between the system performance, i.e., BER used in this paper, and phased DM array excitation configurations, i.e., Phase $e_{m n}$ in Fig. 2, is set up. As a consequence, optimization algorithms can be used to synthesize and optimize the phased DM transmitter arrays by minimizing the value of the cost function, (4). For simplicity we assume the all array element excitation amplitudes $A m n$ are uniform for each symbol transmitted.

Since this optimization task is a multi-variable $(M \times N$ variables for the structure in Fig. 2) and highly non-linear problem, classical optimization algorithms, such as quasiNewton methods and interior point methods, which rely on gradient information, often lead to a local optimal solution. To better attempt to achieve global convergence, population-based optimization approaches are preferred, e.g., Genetic Algorithm (GA) and PSO. We adopt a PSO algorithm for the phased DM array synthesis in this paper since generally it is more computationally efficient than the GA [11].

PSO is a relatively recent heuristic search method that is based on the idea of collaborative behaviour and swarming in biological populations [12], [13]. It depends on information sharing among their population members to enhance their search processes using a combination of deterministic and probabilistic rules.

The PSO algorithmic steps required for a synthesis of a fourelement one dimensional (1-D) phased DM array modulated for QPSK are now briefly presented below,

a) Generate a large number of particles, 1000 in this paper, in the search space. The search region is a 16-dimensional $(M \times N)$ space with each dimension ranging from $0^{\circ}$ to $360^{\circ}$. Each particle locates at a random position $x_{i}(i=1,2, \ldots$, $1000)$ with a coordinate Phase mn $_{\text {for the }}(m \times n)^{\text {th }}$ dimension. By (4) we can find the best global particle position of the entire swarm associated with the minimum value of the cost function, and assign it to a variable $g$, and initializing each particle's best position $p_{\text {i }}$ to its initial position $x_{i}$.

b) Initialize a random velocity $v_{i}$ for each particle. Each dimension of $v_{i}$ is uniformly distributed within 100 to 300 . This range is carefully chosen to balance a tradeoff between convergence performance and best solution quality.

c) Update each velocity $v_{i}$ via (6),

$$
v_{i} \leftarrow v_{i}+\varphi_{p} \cdot r_{p} \cdot\left(p_{i}-x_{i}\right)+\varphi_{g} \cdot r_{g} \cdot\left(g-x_{i}\right)
$$

$r_{p}$ and $r_{g}$ are random variables, uniformly distributed from 0 to 1 . Both $\varphi_{p}$ and $\varphi_{g}$, named as acceleration constants associated with best particle position and best global position, are set to 2 .

d) Update each particle's position $x_{i}$, (7), Wraparound boundary condition is adopted.

$$
x_{i} \leftarrow x_{i}+v_{i}
$$

e) Calculate the value of cost functions for each updated $x_{i}$, and assign $p_{\mathrm{i}}$ and $g$ with the best known particle position and global position, respectively, associated with the minimum value of the cost function.

f) Iterate (b) to (e) for 5000 times, then $g$ holds the best found solution, i.e., the synthesized Phase ${ }_{m n}$ for DM arrays.

Following the optimization steps described above and using the weights in (5), a four-element half-wavelength spaced 1-D phased DM array for QPSK modulation is synthesized for boresight $\left(90^{\circ}\right)$ communication. The phase shifter configurations are presented in Table I, and the calculated BER spatial distribution is illustrated in Fig. 4. Here the conventional phased array used as a reference comparison refers to an array with uniform excitation magnitude and progressive phase. The excitation magnitude is normalized in order to scale BER at the selected communication direction $\left(90^{\circ}\right)$ to the same value $10^{-8}$. It can be observed in Fig. 4 that the synthesized DM transmitter array is able to produce much narrower BER beam and less notable sidelobes than those obtained for the conventional array, which leads to enhanced security performance. Similar observations can be obtained in cases for other spatial direction communications, e.g., the BER distribution for $30^{\circ}$ direction secure communication is illustrated in Fig. 5 with synthesized Phase $_{m n}$ listed in Table II.

\begin{tabular}{|c|c|c|c|c|}
\hline \multicolumn{5}{|c|}{ Phased DM transmitter array } \\
\hline Phase $_{\text {mn }}$ & $n=1$ & $n=2$ & $n=3$ & $n=4$ \\
\hline$m=1$ (Symbol '11') & $276^{\circ}$ & $60^{\circ}$ & $81^{\circ}$ & $63^{\circ}$ \\
\hline$m=2$ (Symbol '01') & $332^{\circ}$ & $344^{\circ}$ & $344^{\circ}$ & $196^{\circ}$ \\
\hline$m=3$ (Symbol '00') & $243^{\circ}$ & $273^{\circ}$ & $238^{\circ}$ & $179^{\circ}$ \\
\hline$m=4$ (Symbol '10') & $142^{\circ}$ & $147^{\circ}$ & $168^{\circ}$ & $122^{\circ}$ \\
\hline \multicolumn{5}{|c|}{ Conventional phased array } \\
\hline Phase $_{m n}$ & $n=1$ & $n=2$ & $n=3$ & $n=4$ \\
\hline$(m=1,2,3,4)$ & $0^{\circ}$ & $0^{\circ}$ & $0^{\circ}$ & $0^{\circ}$ \\
\hline
\end{tabular}

TABLE I. SyNTHESIZED PHASE SHIFTER VALUES FOR BORESIGHT SECURE COMMUNICATION

TABLE II. SyNTHESIZED PHASE SHIFTER VALUES FOR 30 SECURE COMMUNICATION

\begin{tabular}{|c|c|c|c|c|}
\hline \multicolumn{5}{|c|}{ Phased DM transmitter array } \\
\hline Phase $_{\boldsymbol{m} \boldsymbol{n}}$ & $\boldsymbol{n}=\mathbf{1}$ & $\boldsymbol{n}=\mathbf{2}$ & $\boldsymbol{n}=\mathbf{3}$ & $\boldsymbol{n}=\mathbf{4}$ \\
\hline $\boldsymbol{m}=\mathbf{1}$ (Symbol '11') & $84^{\circ}$ & $194^{\circ}$ & $130^{\circ}$ & $240^{\circ}$ \\
\hline $\boldsymbol{m}=\mathbf{2}$ (Symbol '01') $^{\text {(Symbol }}$ & $91^{\circ}$ & $184^{\circ}$ & $267^{\circ}$ & $112^{\circ}$ \\
\hline
\end{tabular}




\begin{tabular}{|c|c|c|c|c|}
\hline$m=3$ (Symbol '00’) & $334^{\circ}$ & $139^{\circ}$ & $192^{\circ}$ & $344^{\circ}$ \\
\hline$m=4$ (Symbol '10') & $231^{\circ}$ & $58^{\circ}$ & $121^{\circ}$ & $236^{\circ}$ \\
\hline \multicolumn{5}{|c|}{ Conventional phased array } \\
\hline Phase $_{m n}$ & $n=1$ & $n=2$ & $n=3$ & $n=4$ \\
\hline$(m=1,2,3,4)$ & $0^{\circ}$ & $156^{\circ}$ & $312^{\circ}$ & $108^{\circ}$ \\
\hline
\end{tabular}

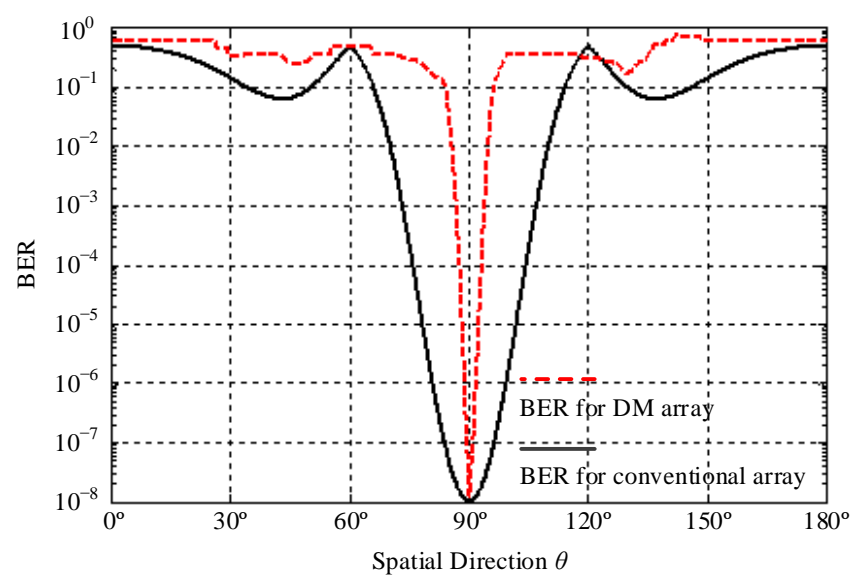

Fig. 4. BER spatial distributions for the synthesized DM array in Table I and the conventional array for boresigh secure communications. SNR is set to 15 $\mathrm{dB}$ along $90^{\circ}$ spatial direction.

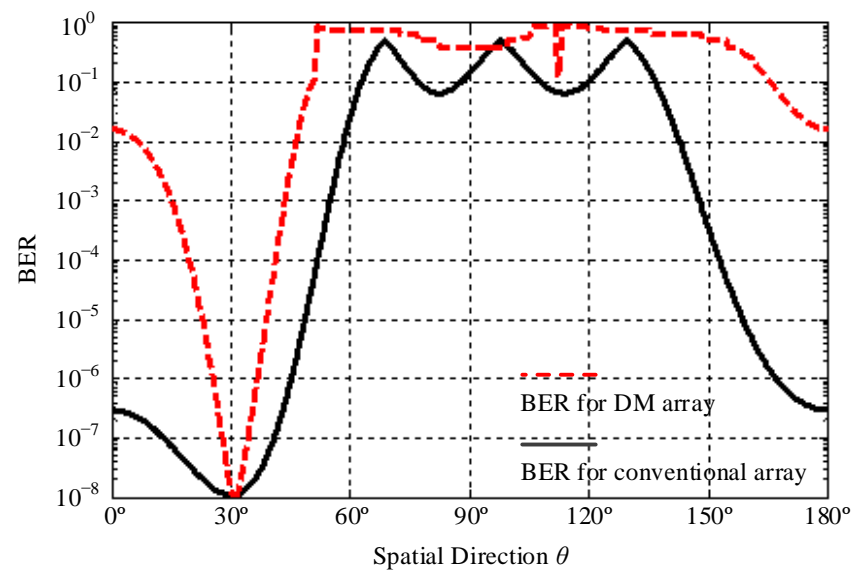

Fig. 5. BER spatial distributions for the synthesized DM array in Table II and the conventional array for $30^{\circ}$ direction secure communications. SNR is set to $15 \mathrm{~dB}$ along $30^{\circ}$ spatial direction.

\section{CONCLUSIONS}

By establishing a link between bit error rate (BER) performance of a directional modulation (DM) system and the DM transmitter array configurations, a cost function was designed. The PSO algorithm was applied to DM transmitter synthesis by minimizing the value of the cost function. The synthesized DM transmitter arrays for QPSK modulation showed enhanced security performance with regard to BER beamwidths and maximum sidelobe levels, when compared with those that could be obtained when conventional phased array transmitters were used.

\section{ACKNOWLEDGMENT}

The authors thank Mrs Yi He for useful discussions.

\section{REFERENCES}

[1] X. Li, J. Hwu and E. P. Ratazzi, "Using antenna array redundancy and channel diversity for secure wireless transmissions," Journal of Communications, Vol. 2, No. 3, pp. 24-32, May 2007.

[2] Y. Hwang and H. C. Papadopoulos, "Physical-layer secrecy in AWGN via a class of chaotic DS/SS systems: analysis and design,” IEEE Trans. Signal Processing, Vol. 52, No. 9, pp. 2637-2649, Sept. 2004.

[3] Yi-Sheng Shiu, Shih-Yu Chang, Hsiao-Chun Wu, S.C.-. Huang and Hsiao-Hwa Chen, "Physical layer security in wireless networks: a tutorial, ”Wireless Communications, IEEE, Vol. 18, pp. 66-74, 2011.

[4] C. M. Elam and D. A. Leavy, "Secure communication using array transmitter,” U. S. Patent 6,275,679, August 14, 2001.

[5] A Babakhani, D. B. Rutledge and A. Hajimiri, "Transmitter architectures based on near-field direct antenna modulation," Solid-State Circuits, IEEE Journal of, Vol. 43, pp. 2674-2692, 2008.

[6] A. Babakhani, D. Rutledge and A. Hajimiri, "Near-field direct antenna modulation,” Microwave Magazine, IEEE, Vol.10, pp. 36-46, 2009.

[7] M. P. Daly and J. T. Bernhard, "Directional modulation technique for phased arrays,” Antennas and Propagation, IEEE Transactions on, Vol. 57, pp. 2633-2640, 2009.

[8] M. P. Daly and J. T. Bernhard, "Beamsteering in pattern reconfigurable arrays using directional modulation,” Antennas and Propagation, IEEE Transactions on, Vol. 58, pp. 2259-2265, 2010.

[9] Hong Zhe Shi and A. Tennant, "An experimental two element array configured for directional antenna modulation," in Antennas and Propagation (EUCAP), 2012 6th European Conference on, pp. 16241626, 2012.

[10] R.A. Shafik, S. Rahman and AHM Razibul Islam, “On the extended relationships among EVM, BER and SNR as performance metrics,” in Electrical and Computer Engineering, 2006. ICECE '06. International Conference on, pp. 408-411, 2006.

[11] R. Hassan, B. K. Cohanim, O. D. Weck, G. Venter, “A comparison of particle swarm optimization and the genetic algorithm,” In Proceedings of the 46th AIAA/ASME/ASCE/AHS/ASC structures, structural dynamics and materials conference, Austin, TX, 2005.

[12] J. Kennedy and R. Eberhart, "Particle swarm optimization,” in Neural Networks, 1995. Proceedings, IEEE International Conference on, pp. 1942-1948 vol.4, 1995.

[13] Y. Shi and R. Eberhart, "A modified particle swarm optimizer," in Evolutionary Computation Proceedings, 1998. IEEE World Congress on Computational Intelligence, The 1998 IEEE International Conference on, pp. 69-73, 1998. 